\title{
Convergent Analysis of Energy Conservative Algorithm for the Nonlinear Schrödinger Equation
}

\author{
Lv Zhong-Quan, ${ }^{1,2,3}$ Gong Yue-Zheng, ${ }^{3}$ and Wang Yu-Shun ${ }^{3}$ \\ ${ }^{1}$ College of Science, Nanjing University of Aeronautics and Astronautics, Nanjing 210016, China \\ ${ }^{2}$ College of Science, Nanjing Forestry University, Nanjing 210037, China \\ ${ }^{3}$ Jiangsu Key Laboratory for NSLSCS, School of Mathematical Sciences, Nanjing Normal University, Nanjing 210097, China
}

Correspondence should be addressed to Gong Yue-Zheng; gyz8814@aliyun.com

Received 26 September 2014; Accepted 27 February 2015

Academic Editor: Slim Choura

Copyright (C) 2015 Lv Zhong-Quan et al. This is an open access article distributed under the Creative Commons Attribution License, which permits unrestricted use, distribution, and reproduction in any medium, provided the original work is properly cited.

\begin{abstract}
Using average vector field method in time and Fourier pseudospectral method in space, we obtain an energy-preserving scheme for the nonlinear Schrödinger equation. We prove that the proposed method conserves the discrete global energy exactly. A deduction argument is used to prove that the numerical solution is convergent to the exact solution in discrete $L_{2}$ norm. Some numerical results are reported to illustrate the efficiency of the numerical scheme in preserving the energy conservation law.
\end{abstract}

\section{Introduction}

The nonlinear Schrödinger (NLS) equation describes a wide range of physical phenomena, such as hydrodynamics, plasma physics, nonlinear optics, self-focusing in laser pulses, propagation of heat pulses in crystals, and description of the dynamics of Bose-Einstein condensate at extremely low temperature $[1,2]$. It plays an essential role in mathematical and physical context, and more and more focus is concentrated upon its numerical solvers in recent years [3,4]. For the NLS equation, construction and theoretical analysis of numerical algorithms have achieved fruitful results [5-14].

The general form of the NLS equation with the initial value and the periodic boundary condition is

$$
\begin{gathered}
i \psi_{t}+\psi_{x x}+a|\psi|^{2} \psi=0, \\
\psi(0, t)=\psi(2 \pi, t),
\end{gathered}
$$

where $a$ is a real parameter. Now using $\psi=p+i q$, we can rewrite (1) as a pair of real-valued equations as follows:

$$
\begin{aligned}
& p_{t}+q_{x x}+a\left(p^{2}+q^{2}\right) q=0, \\
& q_{t}-p_{x x}-a\left(p^{2}+q^{2}\right) p=0 .
\end{aligned}
$$

Equations (2) can be expressed in the Hamiltonian form. Consider

$$
\frac{d z}{d t}=J \frac{\delta H(z)}{\delta z},
$$

where $z=(p, q)^{T} \in \mathbb{R}^{2}$ and the Hamiltonian function, which is system energy, is

$$
\begin{gathered}
H(z)=\int_{0}^{2 \pi} \frac{1}{2}\left(p_{x}^{2}+q_{x}^{2}-\frac{a}{2}\left(p^{2}+q^{2}\right)^{2}\right) d x \\
J=\left(\begin{array}{cc}
0 & 1 \\
-1 & 0
\end{array}\right) .
\end{gathered}
$$

The NLS equation (1) admits the energy conservation law. Consider

$$
\varepsilon(t)=\int_{0}^{2 \pi}\left(\frac{a}{4}|\psi|^{4}-\frac{1}{2}\left|\psi_{x}\right|^{2}\right) d x=\varepsilon(0) .
$$

Quispel and McLaren [15] proposed the average vector field (AVF) method, which is a second-order energypreserving method, and they also provided the corresponding high-order method which is of fourth-order accuracy. The second-order energy-preserving method has been applied 
to solve the partial differential equation [16]. However, to our knowledge, the current papers are most concentrated on construction of energy-preserving scheme, and very few papers discussed convergent analysis of the energypreserving scheme. In this paper, we develop an energy conservative algorithm for the NLS equation by using AVF method in time and Fourier pseudospectral method in space and analyze the proposed method.

The paper is organized as follows. In Section 2, a new conservative scheme is proposed for the NLS equation. We prove that the method preserves the energy conservation law. In Section 3, a deduction argument is used to prove that the numerical solution is convergent to the exact solution in discrete $L_{2}$ norm. The solitary wave behaviors for the NLS equation are simulated by the new scheme in Section 4 . In Section 5 , it is devoted to the conclusions.

\section{Construction of Conservative Algorithm for the NLS Equation}

In this section, we apply the Fourier pseudospectral method in space and the AVF method in time to construct an energypreserving algorithm for the NLS equation.

One usually uses second-order Fourier spectral differentiation matrix $D_{2}$ to approximate the second-order differential operator $\partial_{x x}$. For the ordinary differential equation $u_{x x}=f$, we set $u_{x}=v$ and $v_{x}=f$. Applying the Fourier pseudospectral method to the two equations leads to $D_{1} u=v$ and $D_{1} v=f$. Eliminating vector $v$ gives $D_{1}^{2} u=$ $f$. In this work, we use $D_{1}^{2} u$ to approximate $u_{x x}$ instead of $D_{2} u$ and obtain the corresponding Fourier pseudospectral semidiscretization for the NLS equation (1) as follows:

$$
i \frac{d}{d t} \psi_{j}+\left(D_{1}^{2} \Psi\right)_{j}+a\left|\psi_{j}\right|^{2} \psi_{j}=0, \quad j=0,1,2, \ldots, N-1
$$

where $\Psi=\left(\psi_{0}, \psi_{1}, \psi_{2}, \ldots, \psi_{N-1}\right)^{T}$. Equations (6) can be rewritten as

$$
\begin{aligned}
& \frac{d}{d t} p_{j}+\left(D_{1}^{2} \mathbf{q}\right)_{j}+a\left(p_{j}^{2}+q_{j}^{2}\right) q_{j}=0, \\
& \frac{d}{d t} q_{j}-\left(D_{1}^{2} \mathbf{p}\right)_{j}-a\left(p_{j}^{2}+q_{j}^{2}\right) p_{j}=0,
\end{aligned}
$$

where $\mathbf{p}=\left(p_{0}, p_{1}, \ldots, p_{N-1}\right)^{T}$ and $\mathbf{q}=\left(q_{0}, q_{1}, \ldots, q_{N-1}\right)^{T}$. Since $D_{1}^{2}$ is symmetric, (7) is regarded as a Hamiltonian system with Hamiltonian. Consider

$$
H(\mathbf{p}, \mathbf{q})=\frac{1}{2}\left(\mathbf{p}^{T} D_{1}^{2} \mathbf{p}+\mathbf{q}^{T} D_{1}^{2} \mathbf{q}\right)+\frac{a}{4} \sum_{j=0}^{N-1}\left(\left(p_{j}\right)^{2}+\left(q_{j}\right)^{2}\right)^{2}
$$

Now we discretize (6) with respect to time by the AVF method and obtain

$$
\begin{aligned}
i \delta_{t} \psi_{j}^{n}+ & \left(D_{1}^{2} A_{t} \Psi^{n}\right)_{j} \\
+ & a\left(A_{t}\left(\left|\psi_{j}^{n}\right|^{2}\right) A_{t} \psi_{j}^{n}\right. \\
& \left.+\frac{1}{6}\left(\psi_{j}^{n+1}-\psi_{j}^{n}\right)\left(\psi_{j}^{n+1} p_{j}^{n}-\psi_{j}^{n} p_{j}^{n+1}\right)\right)=0,
\end{aligned}
$$

where $\delta_{t} \psi_{j}^{n}=\left(\psi_{j}^{n+1}-\psi_{j}^{n}\right) / \tau, A_{t} \psi_{j}^{n}=\left(\psi_{j}^{n+1}+\psi_{j}^{n}\right) / 2$, and $p_{j}^{n}=\operatorname{Re}\left(\psi_{j}^{n}\right)=\left(\psi_{j}^{n}+\overline{\psi_{j}^{n}}\right) / 2$. Obviously, scheme (9) can be reformed as a vector form. Consider

$$
\begin{gathered}
i \delta_{t} \Psi^{n}+D_{1}^{2} A_{t} \Psi^{n} \\
+a\left(A_{t}\left(\left|\Psi^{n}\right|^{2}\right) \cdot A_{t} \Psi^{n}+\frac{1}{6}\left(\Psi^{n+1}-\Psi^{n}\right)\right. \\
\left.\cdot\left(\Psi^{n+1} \cdot P^{n}-\Psi^{n} \cdot P^{n+1}\right)\right)=0,
\end{gathered}
$$

where $\left|\Psi^{n}\right|^{2}=\left|\Psi^{n}\right| \cdot\left|\Psi^{n}\right|$ and “.” denotes point multiplication between vectors; that is,

$$
\mathbf{p}^{n} \cdot \mathbf{q}^{n}=\left(p_{0}^{n} q_{0}^{n}, p_{1}^{n} q_{1}^{n}, p_{2}^{n} q_{2}^{n}, \ldots, p_{N-1}^{n} q_{N-1}^{n}\right)^{T} .
$$

Equations (9) can also be rewritten as

$$
\begin{aligned}
& \frac{p_{j}^{n+1}-p_{j}^{n}}{\tau}+\left(D_{1}^{2} \mathbf{q}^{n+1 / 2}\right)_{j} \\
& +a\left(\frac{1}{4}\left(q_{j}^{n+1}+q_{j}^{n}\right)\right. \\
& \cdot\left(\left(p_{j}^{n+1}\right)^{2}+\left(p_{j}^{n}\right)^{2}+\left(q_{j}^{n+1}\right)^{2}+\left(q_{j}^{n}\right)^{2}\right) \\
& \left.+\frac{1}{6}\left(p_{j}^{n+1}-p_{j}^{n}\right)\left(q_{j}^{n+1} \cdot p_{j}^{n}-q_{j}^{n} \cdot p_{j}^{n+1}\right)\right)=0, \\
& \frac{q_{j}^{n+1}-q_{j}^{n}}{\tau}-\left(D_{1}^{2} \mathbf{p}^{n+1 / 2}\right)_{j} \\
& -a\left(\frac{1}{4}\left(p_{j}^{n+1}+p_{j}^{n}\right)\right. \\
& \quad\left(\left(p_{j}^{n+1}\right)^{2}+\left(p_{j}^{n}\right)^{2}+\left(q_{j}^{n+1}\right)^{2}+\left(q_{j}^{n}\right)^{2}\right) \\
& \left.+\frac{1}{6}\left(q_{j}^{n+1}-q_{j}^{n}\right)\left(p_{j}^{n+1} \cdot q_{j}^{n}-p_{j}^{n} \cdot q_{j}^{n+1}\right)\right)=0 .
\end{aligned}
$$

Next, we prove that scheme (10) conserves the discrete total energy. Let $X_{N}=\left\{u \mid u=\left(u_{0}, u_{1}, \ldots, u_{N-1}\right)\right\} \subseteq \mathbb{C}^{N}$ and define discrete inner product and discrete $L_{2}$ norm over $X_{N}$ as

$$
(u, v)_{N}=h \sum_{j=0}^{N-1} u_{j} \overline{v_{j}}, \quad\|u\|_{N}=(u, u)_{N}^{1 / 2}
$$


Theorem 1. With periodic boundary condition $\psi(0, t)=$ $\psi(2 \pi, t)$, scheme (10) possesses the discrete global energy conservation law; namely,

$$
\varepsilon^{n+1}=\varepsilon^{n}=\cdots=\varepsilon^{1}=\varepsilon^{0},
$$

where $\varepsilon^{n}=(a / 4)\left\|\Psi^{n}\right\|_{N, 4}^{4}-(1 / 2)\left\|D_{1} \Psi^{n}\right\|_{N}^{2}$ and $\left\|\Psi^{n}\right\|_{N, 4}^{4}=$ $\left(\left|\Psi^{n}\right|^{2},\left|\Psi^{n}\right|^{2}\right)_{N}$.

Proof. Taking the inner product of (10) with $\Psi^{n+1}-\Psi^{n}$ yields

$$
\begin{gathered}
i\left(\delta_{t} \Psi^{n}, \Psi^{n+1}-\Psi^{n}\right)_{N}+\left(D_{1}^{2} A_{t} \Psi^{n}, \Psi^{n+1}-\Psi^{n}\right)_{N} \\
+a\left(A_{t}\left(\left|\Psi^{n}\right|^{2}\right) \cdot A_{t} \Psi^{n}+\frac{1}{6}\left(\Psi^{n+1}-\Psi^{n}\right)\right. \\
\left.\cdot\left(\Psi^{n+1} \cdot P^{n}-\Psi^{n} \cdot P^{n+1}\right), \Psi^{n+1}-\Psi^{n}\right)_{N}=0 .
\end{gathered}
$$

The first term becomes

$$
\frac{i}{\tau}\left(\Psi^{n+1}-\Psi^{n}, \Psi^{n+1}-\Psi^{n}\right)_{N}=\frac{i}{\tau}\left\|\Psi^{n+1}-\Psi^{n}\right\|_{N}^{2},
$$

which is purely imaginary. The second term can be reduced to

$$
\begin{aligned}
\frac{1}{2}\left(D_{1}^{2}\left(\Psi^{n+1}+\Psi^{n}\right), \Psi^{n+1}-\Psi^{n}\right)_{N} \\
=-\frac{1}{2}\left(\left\|D_{1} \Psi^{n+1}\right\|_{N}^{2}-\left\|D_{1} \Psi^{n}\right\|_{N}^{2}\right) \\
+i \operatorname{Im}\left(D_{1} \Psi^{n+1}, D_{1} \Psi^{n}\right)_{N} .
\end{aligned}
$$

Noticing that $P^{n}=\left(\Psi^{n}+\overline{\Psi^{n}}\right) / 2$, we have

$$
\begin{aligned}
a\left(A_{t}\left(\left|\Psi^{n}\right|^{2}\right) \cdot A_{t} \Psi^{n}+\frac{1}{6}\left(\Psi^{n+1}-\Psi^{n}\right)\right. & \left.\cdot\left(\Psi^{n+1} \cdot P^{n}-\Psi^{n} \cdot P^{n+1}\right), \Psi^{n+1}-\Psi^{n}\right)_{N} \\
=a & \left\{\frac{1}{4}\left(\left\|\Psi^{n+1}\right\|_{N, 4}^{4}-\left\|\Psi^{n}\right\|_{N, 4}^{4}\right)\right. \\
& -\frac{i}{2}\left(\left|\Psi^{n+1}\right|^{2}+\left|\Psi^{n}\right|^{2}, \operatorname{Im}\left(\Psi^{n+1} \cdot \overline{\Psi^{n}}\right)\right)_{N} \\
+ & \left.\frac{i}{6}\left(\operatorname{Im}\left(\Psi^{n+1} \cdot \overline{\Psi^{n}}\right),\left|\Psi^{n+1}-\Psi^{n}\right|^{2}\right)_{N}\right\} .
\end{aligned}
$$

Therefore, the real part of (15) is

$$
\begin{aligned}
-\frac{1}{2}( & \left.\left\|D_{1} \Psi^{n+1}\right\|_{N}^{2}-\left\|D_{1} \Psi^{n}\right\|_{N}^{2}\right) \\
& +\frac{a}{4}\left(\left\|\Psi^{n+1}\right\|_{N, 4}^{4}-\left\|\Psi^{n}\right\|_{N, 4}^{4}\right)=0 .
\end{aligned}
$$

So (19) gives the energy conservation law (14).

\section{Convergence Analysis}

Let $I=[0,2 \pi], L^{2}(I)$ with the inner product $(\cdot, \cdot)$ and the norm $\|\cdot\|$. For any positive integer $r$, the seminorm and the norm of $H^{r}(I)$ are denoted by $|\cdot|_{r}$ and $\|\cdot\|_{r}$, respectively. Let $C_{(p)}^{\infty}(I)$ be the set of infinitely differentiable functions with period $2 \pi$, defined on $\mathbb{R}$. $H_{(p)}^{r}(I)$ is the closure of $C_{(p)}^{\infty}(I)$ in $H^{r}(I)$. In this section, let $C$ be a generic positive constant which may be dependent on the regularity of exact solution and the initial data but independent of the time step $\tau$ and the grid size $h$.

For even $N$, set

$$
\begin{gathered}
V_{N}=\left\{u\left|u(x)=\sum_{|k| \leq N / 2} \widehat{u}_{k} e^{i k x},{\overline{u_{k}}}_{k} \widehat{u}_{-k},\right| k \mid \leq \frac{N}{2}\right\}, \\
V_{N}^{\prime \prime}=\left\{u \mid u(x)=\sum_{|k| \leq N / 2}{ }^{\prime \prime} \widehat{u}_{k} e^{i k x}, \overline{\widehat{u}_{k}}=\widehat{u}_{-k},\right. \\
\left.|k| \leq \frac{N}{2}, \widehat{u}_{N / 2}=\widehat{u}_{-N / 2}\right\},
\end{gathered}
$$

where the summation $\Sigma^{\prime \prime}$ is defined by

$$
\sum_{|k| \leq N / 2}^{\prime \prime} \psi_{k}=\frac{1}{2} \psi_{-N / 2}+\sum_{|k|<N / 2} \psi_{k}+\frac{1}{2} \psi_{N / 2} .
$$

It is obviously that $V_{N}^{\prime \prime} \subseteq V_{N}$. Denote the orthogonal projection operator $P_{N}: L^{2}(I) \rightarrow V_{N}$ and the interpolation operator $I_{N}: L^{2}(I) \rightarrow V_{N}^{\prime \prime}$. Note that $P_{N}$ and $I_{N}$ satisfy the following properties:

(1) $P_{N} \partial_{x} u=\partial_{x} P_{N} u, I_{N} \partial_{x} u \neq \partial_{x} I_{N} u$;

(2) $\left(P_{N-2} u, v\right)_{N}=\left(P_{N-2} u, v\right), \forall v \in V_{N}$;

(3) $P_{N} u=u, \forall u \in V_{N} ; I_{N} u=u, \forall u \in V_{N}^{\prime \prime}$.

Lemma 2. For $u \in V_{N}^{\prime \prime},\|u\| \leq\|u\|_{N} \leq \sqrt{2}\|u\|$.

Lemma 3 (see [17]). If $0 \leq l \leq r$ and $u \in H_{(p)}^{r}(I)$, then

$$
\begin{gathered}
\left\|P_{N} u-u\right\|_{l} \leq C N^{l-r}|u|_{r}, \\
\left\|P_{N} u\right\|_{l} \leq C\|u\|_{l},
\end{gathered}
$$

and if $r>1 / 2$, then

$$
\begin{gathered}
\left\|I_{N} u-u\right\|_{l} \leq C N^{l-r}|u|_{r}, \\
\left\|I_{N} u\right\|_{l} \leq C\|u\|_{l} .
\end{gathered}
$$

Lemma 4. Suppose $u^{*}=P_{N-2} u, u \in H_{(p)}^{r}(I)$, and $r>1 / 2$; then $\left\|u^{*}-u\right\|_{N} \leq C N^{-r}|u|_{r}$.

Proof. According to Lemmas 2 and 3, we have

$$
\begin{aligned}
\left\|u^{*}-u\right\|_{N} & =\left\|I_{N}\left(u^{*}-u\right)\right\|_{N} \\
& =\left\|u^{*}-I_{N} u\right\|_{N} \\
& \leq \sqrt{2}\left\|u^{*}-I_{N} u\right\| \\
& \leq \sqrt{2}\left(\left\|u^{*}-u\right\|+\left\|u-I_{N} u\right\|\right) \\
& \leq C N^{-r}|u|_{r} .
\end{aligned}
$$


Lemma 5 (Gronwall's inequality [18]). Suppose that the discrete function $\left\{w^{n} \mid n=0,1,2, \ldots, M ; M \tau=T\right\}$ satisfies the following inequality:

$$
w^{n}-w^{n-1} \leq A \tau w^{n}+B \tau w^{n-1}+C_{n} \tau,
$$

where $A, B$, and $C_{n}(n=0,1,2, \ldots, M)$ are nonnegative constants. Then

$$
\max _{1 \leq n \leq M}\left|w^{n}\right| \leq\left(w^{0}+\sum_{l=1}^{M} C_{l}\right) e^{2(A+B) T},
$$

where $\tau$ is sufficiently small, such that $(A+B) \tau \leq(M-$ $1) / 2 M,(M>1)$.

An equivalent form of full-discrete Fourier pseudospectral scheme (12) is to find $\left(p_{c}^{n}, q_{c}^{n}\right)^{T} \in\left(V_{N}^{\prime \prime}\right)^{2}$, so that, for any $\Phi^{n}=\left(\Phi_{1}^{n}, \Phi_{2}^{n}\right)^{T} \in\left(V_{N}^{\prime \prime}\right)^{2}$, then

$$
\begin{gathered}
\left(\frac{p_{c}^{n+1}-p_{c}^{n}}{\tau}, \Phi_{1}^{n}\right)_{N}-\left(\partial_{x} q_{c}^{n+1 / 2}, \partial_{x} \Phi_{1}^{n}\right)_{N} \\
\quad+\left(f\left(p_{c}^{n}, p_{c}^{n+1}, q_{c}^{n}, q_{c}^{n+1}\right), \Phi_{1}^{n}\right)_{N}=0, \\
\left(\frac{q_{c}^{n+1}-q_{c}^{n}}{\tau}, \Phi_{2}^{n}\right)_{N}+\left(\partial_{x} p_{c}^{n+1 / 2}, \partial_{x} \Phi_{2}^{n}\right)_{N} \\
-\left(f\left(q_{c}^{n}, q_{c}^{n+1}, p_{c}^{n}, p_{c}^{n+1}\right), \Phi_{2}^{n}\right)_{N}=0,
\end{gathered}
$$

where

$$
\begin{aligned}
f(x, y, u, v) & \\
=a( & \frac{1}{4}(u+v) \cdot\left(x^{2}+y^{2}+u^{2}+v^{2}\right) \\
& \left.+\frac{1}{6}(y-x)(v \cdot x-u \cdot y)\right) .
\end{aligned}
$$

Proposed scheme (10) conserves the energy exactly, which can be regarded as the energy stable algorithm. So we assume that the numerical solution is bounded; that is,

$$
\max _{1 \leq n \leq N}\left\|p_{c}^{n}\right\|_{\infty} \leq C, \quad \max _{1 \leq n \leq N}\left\|q_{c}^{n}\right\|_{\infty} \leq C .
$$

Theorem 6. Suppose that the exact solutions $p, q \in$ $H^{1}\left(0, T ; H_{p}^{r}(I)\right) \cap H^{3}\left(0, T ; L^{2}(I)\right), r>1 / 2$, and $\tau$ are small enough; then the solution of full-discrete Fourier pseudospectral scheme (12) converges to the solution of problems (3) with order $\mathrm{O}\left(\mathrm{N}^{-r}+\tau^{2}\right)$ in discrete $L_{2}$ norm.

Proof. Let $p^{*}=P_{N-2} p$ and $q^{*}=P_{N-2} q$; we have from (2)

$$
\begin{aligned}
& p_{t}^{*}+q_{x x}^{*}+P_{N-2}\left[a\left(p^{2}+q^{2}\right) q\right]=0, \\
& q_{t}^{*}-p_{x x}^{*}-P_{N-2}\left[a\left(p^{2}+q^{2}\right) p\right]=0,
\end{aligned}
$$

and then

$$
\begin{aligned}
& \frac{p^{* n+1}-p^{* n}}{\tau}+\partial_{x x}\left(q^{*}\right)^{n+1 / 2}+P_{N-2}\left[a\left(p^{2}+q^{2}\right) q\right]^{n+1 / 2} \\
& =\frac{p^{* n+1}-p^{* n}}{\tau}-\left(p_{t}^{*}\right)^{n+1 / 2} \triangleq \xi_{1}^{n},
\end{aligned}
$$

$$
\begin{aligned}
& \frac{q^{* n+1}-q^{* n}}{\tau}-\partial_{x x}\left(p^{*}\right)^{n+1 / 2}-P_{N-2}\left[a\left(p^{2}+q^{2}\right) p\right]^{n+1 / 2} \\
& =\frac{q^{* n+1}-q^{* n}}{\tau}-\left(q_{t}^{*}\right)^{n+1 / 2} \triangleq \xi_{2}^{n},
\end{aligned}
$$

where $\left(p^{*}\right)^{n+1 / 2}=\left(p^{* n}+p^{* n+1}\right) / 2$ and so forth. Using Taylor's expansion, we obtain

$$
\left\|\xi_{1}^{n}\right\|_{N} \leq C \tau^{2}, \quad\left\|\xi_{2}^{n}\right\|_{N} \leq C \tau^{2} .
$$

For any $\Phi^{n}=\left(\Phi_{1}^{n}, \Phi_{2}^{n}\right)^{T} \in\left(V_{N}^{\prime \prime}\right)^{2},(32)$ are equivalent to the following equations:

$$
\begin{aligned}
& \left(\frac{p^{* n+1}-p^{* n}}{\tau}, \Phi_{1}^{n}\right)_{N}+\left(\partial_{x x}\left(q^{*}\right)^{n+1 / 2}, \Phi_{1}^{n}\right)_{N} \\
& \quad+\left(P_{N-2}\left[a\left(p^{2}+q^{2}\right) q\right]^{n+1 / 2}, \Phi_{1}^{n}\right)_{N}=\left(\xi_{1}^{n}, \Phi_{1}^{n}\right)_{N}, \\
& \left(\frac{q^{* n+1}-q^{* n}}{\tau}, \Phi_{2}^{n}\right)_{N}-\left(\partial_{x x}\left(p^{*}\right)^{n+1 / 2}, \Phi_{2}^{n}\right)_{N} \\
& \quad-\left(P_{N-2}\left[a\left(p^{2}+q^{2}\right) p\right]^{n+1 / 2}, \Phi_{2}^{n}\right)_{N}=\left(\xi_{2}^{n}, \Phi_{2}^{n}\right)_{N} .
\end{aligned}
$$

According to $\left(P_{N-2} u, v\right)_{N}=\left(P_{N-2} u, v\right), \forall v \in V_{N}$ and $P_{N} \partial_{x} u=$ $\partial_{x} P_{N} u$, we can deduce

$$
\begin{aligned}
& \left(\frac{p^{* n+1}-p^{* n}}{\tau}, \Phi_{1}^{n}\right)_{N}-\left(\partial_{x}\left(q^{*}\right)^{n+1 / 2}, \partial_{x} \Phi_{1}^{n}\right)_{N} \\
& +\left(P_{N-2}\left[a\left(p^{2}+q^{2}\right) q\right]^{n+1 / 2}, \Phi_{1}^{n}\right)_{N}=\left(\xi_{1}^{n}, \Phi_{1}^{n}\right)_{N}, \\
& \left(\frac{q^{* n+1}-q^{* n}}{\tau}, \Phi_{2}^{n}\right)_{N}+\left(\partial_{x}\left(p^{*}\right)^{n+1 / 2}, \partial_{x} \Phi_{2}^{n}\right)_{N} \\
& -\left(P_{N-2}\left[a\left(p^{2}+q^{2}\right) p\right]^{n+1 / 2}, \Phi_{2}^{n}\right)_{N}=\left(\xi_{2}^{n}, \Phi_{2}^{n}\right)_{N} .
\end{aligned}
$$

Let $\varepsilon^{n}=p^{* n}-p_{c}^{n}$ and $\eta^{n}=q^{* n}-q_{c}^{n}$. Subtracting (27)-(28) from (35)-(36), respectively, we obtain the error equations:

$$
\begin{aligned}
& \left(\frac{\varepsilon^{n+1}-\varepsilon^{n}}{\tau}, \Phi_{1}^{n}\right)_{N}-\left(\partial_{x} \eta^{n+1 / 2}, \partial_{x} \Phi_{1}^{n}\right)_{N} \\
& +\left(P_{N-2}\left[a\left(p^{2}+q^{2}\right) q\right]^{n+1 / 2}\right. \\
& \left.\quad-f\left(p_{c}^{n}, p_{c}^{n+1}, q_{c}^{n}, q_{c}^{n+1}\right), \Phi_{1}^{n}\right)_{N}=\left(\xi_{1}^{n}, \Phi_{1}^{n}\right)_{N}, \\
& \left(\frac{\eta^{n+1}-\eta^{n}}{\tau}, \Phi_{2}^{n}\right)_{N}+\left(\partial_{x} \varepsilon^{n+1 / 2}, \partial_{x} \Phi_{2}^{n}\right)_{N} \\
& -\left(P_{N-2}\left[a\left(p^{2}+q^{2}\right) p\right]^{n+1 / 2}\right. \\
& \left.\quad-f\left(q_{c}^{n}, q_{c}^{n+1}, p_{c}^{n}, p_{c}^{n+1}\right), \Phi_{2}^{n}\right)_{N}=\left(\xi_{2}^{n}, \Phi_{2}^{n}\right)_{N} .
\end{aligned}
$$


We take $\Phi_{1}^{n}=\varepsilon^{n+1 / 2}$ and $\Phi_{2}^{n}=\eta^{n+1 / 2}$, and then

$$
\begin{gathered}
\left(\frac{\varepsilon^{n+1}-\varepsilon^{n}}{\tau}, \frac{\varepsilon^{n+1}+\varepsilon^{n}}{2}\right)_{N}-\left(\partial_{x} \eta^{n+1 / 2}, \partial_{x} \varepsilon^{n+1 / 2}\right)_{N} \\
+\left(G_{1}, \varepsilon^{n+1 / 2}\right)_{N}=\left(\xi_{1}^{n}, \varepsilon^{n+1 / 2}\right)_{N}, \\
\left(\frac{\eta^{n+1}-\eta^{n}}{\tau}, \frac{\eta^{n+1}+\eta^{n}}{2}\right)_{N}+\left(\partial_{x} \varepsilon^{n+1 / 2}, \partial_{x} \eta^{n+1 / 2}\right)_{N} \\
-\left(G_{2}, \eta^{n+1 / 2}\right)_{N}=\left(\xi_{2}^{n}, \eta^{n+1 / 2}\right)_{N},
\end{gathered}
$$

where

$$
\begin{aligned}
& G_{1}=P_{N-2}\left[a\left(p^{2}+q^{2}\right) q\right]^{n+1 / 2}-f\left(p_{c}^{n}, p_{c}^{n+1}, q_{c}^{n}, q_{c}^{n+1}\right), \\
& G_{2}=P_{N-2}\left[a\left(p^{2}+q^{2}\right) p\right]^{n+1 / 2}-f\left(q_{c}^{n}, q_{c}^{n+1}, p_{c}^{n}, p_{c}^{n+1}\right) .
\end{aligned}
$$

Adding (38) and (39), we obtain

$$
\begin{aligned}
\frac{1}{2 \tau} & \left(\left\|\varepsilon^{n+1}\right\|_{N}^{2}+\left\|\eta^{n+1}\right\|_{N}^{2}-\left\|\varepsilon^{n}\right\|_{N}^{2}-\left\|\eta^{n}\right\|_{N}^{2}\right) \\
= & \left(\xi_{1}^{n}, \varepsilon^{n+1 / 2}\right)_{N}+\left(\xi_{2}^{n}, \eta^{n+1 / 2}\right)_{N} \\
& -\left(G_{1}, \varepsilon^{n+1 / 2}\right)_{N}+\left(G_{2}, \eta^{n+1 / 2}\right)_{N} .
\end{aligned}
$$

Using Cauchy-Schwarz inequality, we have

$$
\begin{aligned}
\left|\left(\xi_{1}^{n}, \varepsilon^{n+1 / 2}\right)_{N}\right| & \leq\left\|\xi_{1}^{n}\right\|_{N} \cdot\left\|\varepsilon^{n+1 / 2}\right\|_{N} \\
& \leq \frac{1}{2}\left\|\xi_{1}^{n}\right\|_{N}^{2}+\frac{1}{8}\left\|\varepsilon^{n}+\varepsilon^{n+1}\right\|_{N}^{2} \\
& \leq C \tau^{4}+\frac{1}{4}\left(\left\|\varepsilon^{n}\right\|_{N}^{2}+\left\|\varepsilon^{n+1}\right\|_{N}^{2}\right) .
\end{aligned}
$$

Similarly, we have

$$
\begin{aligned}
\left|\left(\xi_{2}^{n}, \eta^{n+1 / 2}\right)_{N}\right| & \leq C \tau^{4}+\frac{1}{4}\left(\left\|\eta^{n}\right\|_{N}^{2}+\left\|\eta^{n+1}\right\|_{N}^{2}\right), \\
\left|\left(G_{1}, \varepsilon^{n+1 / 2}\right)_{N}\right| & \leq \frac{1}{2}\left\|G_{1}\right\|_{N}^{2}+\frac{1}{4}\left(\left\|\varepsilon^{n}\right\|_{N}^{2}+\left\|\varepsilon^{n+1}\right\|_{N}^{2}\right), \\
\left|\left(G_{2}, \eta^{n+1 / 2}\right)_{N}\right| & \leq \frac{1}{2}\left\|G_{2}\right\|_{N}^{2}+\frac{1}{4}\left(\left\|\eta^{n}\right\|_{N}^{2}+\left\|\eta^{n+1}\right\|_{N}^{2}\right) .
\end{aligned}
$$

Using the triangle inequality, we obtain

$$
\begin{aligned}
& \left\|G_{1}\right\|_{N} \\
& \quad=\left\|P_{N-2}\left[a\left(p^{2}+q^{2}\right) q\right]^{n+1 / 2}-f\left(p_{c}^{n}, p_{c}^{n+1}, q_{c}^{n}, q_{c}^{n+1}\right)\right\|_{N} \\
& \quad \leq\left\|P_{N-2}\left[a\left(p^{2}+q^{2}\right) q\right]^{n+1 / 2}-\left[a\left(p^{2}+q^{2}\right) q\right]^{n+1 / 2}\right\|_{N}
\end{aligned}
$$

$$
\begin{aligned}
& +\left\|\left[a\left(p^{2}+q^{2}\right) q\right]^{n+1 / 2}-f\left(p^{n}, p^{n+1}, q^{n}, q^{n+1}\right)\right\|_{N} \\
& +\left\|f\left(p^{n}, p^{n+1}, q^{n}, q^{n+1}\right)-f\left(p^{* n}, p^{* n+1}, q^{* n}, q^{* n+1}\right)\right\|_{N} \\
& +\left\|f\left(p^{* n}, p^{* n+1}, q^{* n}, q^{* n+1}\right)-f\left(p_{c}^{n}, p_{c}^{n+1}, q_{c}^{n}, q_{c}^{n+1}\right)\right\|_{N} \\
& \triangleq I+I I+I I I+I V .
\end{aligned}
$$

According to Lemma 4, $I \leq C N^{-r}$. Using Taylor's expansion, $I I \leq C \tau^{2}$. Using the inequality

$$
|u \cdot v-\widetilde{u} \cdot \widetilde{v}| \leq|u-\widetilde{u}| \cdot|v|+|\widetilde{u}| \cdot|v-\widetilde{v}|
$$

and Lemma 4, $I I I \leq C N^{-r}$. According to inequality (45) and the boundedness of numerical solution (30), $I V \leq C\left(\left\|\varepsilon^{n}\right\|_{N}+\right.$ $\left.\left\|\varepsilon^{n+1}\right\|_{N}+\left\|\eta^{n}\right\|_{N}+\left\|\eta^{n+1}\right\|_{N}\right)$.

Therefore, we can deduce

$$
\begin{aligned}
\left\|G_{1}\right\|_{N}^{2} \leq & C\left(N^{-2 r}+\tau^{4}\right) \\
& +C\left(\left\|\varepsilon^{n}\right\|_{N}^{2}+\left\|\varepsilon^{n+1}\right\|_{N}^{2}+\left\|\eta^{n}\right\|_{N}^{2}+\left\|\eta^{n+1}\right\|_{N}^{2}\right) .
\end{aligned}
$$

Similarly, we have

$$
\begin{aligned}
\left\|G_{2}\right\|_{N}^{2} \leq & C\left(N^{-2 r}+\tau^{4}\right) \\
& +C\left(\left\|\varepsilon^{n}\right\|_{N}^{2}+\left\|\varepsilon^{n+1}\right\|_{N}^{2}+\left\|\eta^{n}\right\|_{N}^{2}+\left\|\eta^{n+1}\right\|_{N}^{2}\right) .
\end{aligned}
$$

Thus, we obtain

$$
\begin{aligned}
\frac{1}{2 \tau} & \left(\left\|\varepsilon^{n+1}\right\|_{N}^{2}+\left\|\eta^{n+1}\right\|_{N}^{2}-\left\|\varepsilon^{n}\right\|_{N}^{2}-\left\|\eta^{n}\right\|_{N}^{2}\right) \\
\leq & C\left(N^{-2 r}+\tau^{4}\right) \\
& +C\left(\left\|\varepsilon^{n}\right\|_{N}^{2}+\left\|\varepsilon^{n+1}\right\|_{N}^{2}+\left\|\eta^{n}\right\|_{N}^{2}+\left\|\eta^{n+1}\right\|_{N}^{2}\right) .
\end{aligned}
$$

Let $\omega^{n}=\left\|\varepsilon^{n}\right\|_{N}^{2}+\left\|\eta^{n}\right\|_{N}^{2}$, and (48) can be rewritten as

$$
\omega^{n+1}-\omega^{n} \leq C \tau\left(\omega^{n+1}+\omega^{n}\right)+C \tau\left(N^{-2 r}+\tau^{4}\right) .
$$

According to Lemma 5, we have

$$
\omega^{n} \leq\left(\omega^{0}+\tau \sum_{l=1}^{M} C\left(N^{-2 r}+\tau^{4}\right)\right) e^{4 C T} .
$$

According to Lemma 4 and noticing $p_{c}^{0}=p^{0}$ and $q_{c}^{0}=q^{0}$, we have

$$
\omega^{0}=\left\|\varepsilon^{0}\right\|_{N}^{2}+\left\|\eta^{0}\right\|_{N}^{2}=\left\|p^{* 0}-p^{0}\right\|_{N}^{2}+\left\|q^{* 0}-q^{0}\right\|_{N}^{2} \leq C N^{-2 r} .
$$

Therefore, we get

$$
\omega^{n} \leq C\left(N^{-2 r}+\tau^{4}\right) .
$$


Moreover, we have

$$
\begin{gathered}
\left\|p^{* n}-p_{c}^{n}\right\|_{N} \leq C\left(N^{-r}+\tau^{2}\right), \\
\left\|q^{* n}-q_{c}^{n}\right\|_{N} \leq C\left(N^{-r}+\tau^{2}\right) .
\end{gathered}
$$

Using the triangle inequality and Lemma 4, we obtain

$$
\begin{gathered}
\left\|p^{n}-p_{c}^{n}\right\|_{N} \leq\left\|p^{n}-p^{* n}\right\|_{N}+\left\|p^{* n}-p_{c}^{n}\right\|_{N} \leq C\left(N^{-r}+\tau^{2}\right), \\
\left\|q^{n}-q_{c}^{n}\right\|_{N} \leq\left\|q^{n}-q^{* n}\right\|_{N}+\left\|q^{* n}-q_{c}^{n}\right\|_{N} \leq C\left(N^{-r}+\tau^{2}\right) .
\end{gathered}
$$

This completes the proof.

\section{Numerical Experiments}

In this section, we conduct some tentative numerical experiments for this new scheme (10) to verify the theoretical conclusions, including the accuracy, the ability to preserve the first integrals of the nonlinear Schrödinger equation for long-time integration.

First we take the parameter $a=2$. Then, we get the following:

$$
i \psi_{t}+\psi_{x x}+2|\psi|^{2} \psi=0
$$

We consider nonlinear Schrödinger equation (55) with the one-soliton solution as follows:

$$
\psi(x, t)=\operatorname{sech}(x-4 t) \exp \left(2 i\left(x-\frac{3}{2} t\right)\right) .
$$

In order to analyze new scheme (10), the problem is solved in $[-15,15]$ with the initial condition

$$
u(x, 0)=\operatorname{sech}(x) \exp (2 x i)
$$

We take $N=200$ and the time step $\tau=10^{-3}$ for the new scheme (10). We check the ability of this new scheme preserving the first integral which is one of the important criteria to judge numerical schemes. The nonlinear Schrödinger equation with periodic boundary condition has the energy conservation law:

$$
F(\psi)=\int_{0}^{L}\left[\frac{a}{4}|\psi|^{4}-\frac{1}{2}\left|\psi_{x}\right|^{2}\right] d x .
$$

If the approximate solution of $\psi(x, t=j \tau)$ is $\psi^{j}=$ $\left(\psi_{0}, \psi_{1}, \ldots, \psi_{N}\right)^{T}$, then the discrete conservation law $F$ is

$$
F^{h}(\psi)=\sum_{j=1}^{N} \frac{1}{2}\left(\left|\psi_{j}\right|^{4}-\left|D_{1} \Psi\right|_{j}^{2}\right) h .
$$

We define the errors of the discrete conservation law on the $j$ th time level as

$$
\operatorname{Err}_{F}(j \tau)=F^{h}\left(\psi^{j}\right)-F^{h}\left(\psi^{0}\right)
$$

TABLE 1: Time accuracy of new scheme (10) with initial condition (57) $(N=100)$.

\begin{tabular}{lcc}
\hline$\tau$ & $L_{2}$ error & Order \\
\hline 0.004 & $1.1351 e-004$ & - \\
0.002 & $2.8338 e-005$ & 2.0020 \\
0.001 & $7.0510 e-006$ & 2.0068 \\
0.0005 & $1.7305 e-006$ & 2.0266 \\
0.00025 & $4.0445 e-007$ & 2.0972 \\
0.000125 & $9.4398 e-008$ & 2.0991 \\
\hline
\end{tabular}

where $\psi^{j}$ is the numerical solution on the $j$ th time level and $\psi^{0}$ is the discrete initial value. Numerical solutions and exact solutions at different time levels and the changes of the errors between the exact solutions and the numerical solutions and $\operatorname{Err}_{F}$ with time are shown in Figure 1.

The discrete $L^{2}$ norm of complex-valued function $\psi$ is defined as

$$
\|\psi\|_{2}=\left(\sum_{j=1}^{N}\left|\psi_{j}\right|^{2} h\right)^{1 / 2}
$$

We consider that the problem is solved in $[-15,15]$ till time $t=1$ for accuracy test. Note that in Table 1 the spatial error $(N=100)$ is negligible and the error is dominated by the time discretization error. It shows that accuracy of space is very large. Table 1 clearly indicates that new scheme (10) is of second order in time.

We also test our new scheme on the following initial condition $\psi(x, 0)=0.5+0.025 \cos (\mu x)$ with the periodic boundary condition $\psi(0, t)=\psi(4 \sqrt{2} \pi, t)$. We take $L=4 \sqrt{2} \pi$, $\mu=2 \pi / L$. The initial condition is in the vicinity of the homoclinic orbit in [19].

In this case, we also take $N=200$ and the time step $\tau=10^{-3}$ for new scheme (10). The corresponding waveforms at different time levels and the changes of errors of discrete conservation law $F$ with time are showed in Figure 2. We find that the numerical results we presented in the paper show that the new scheme is very robust and stable. Thus, our new scheme provides a new choice for solving the nonlinear Schrödinger equation.

\section{Conclusions}

In this paper, we derive a new method for the nonlinear Schrödinger system. We prove the proposed method preserves the energy conservation law exactly. A deduction argument is used to prove that the numerical solution is second-order convergent to the exact solutions in $\|\cdot\|_{2}$ norm. Some numerical results are reported to illustrate the efficiency of the numerical scheme in preserving the energy conservation laws. Therefore, it will be a good choice for solving the nonlinear Schrödinger equation computation. 


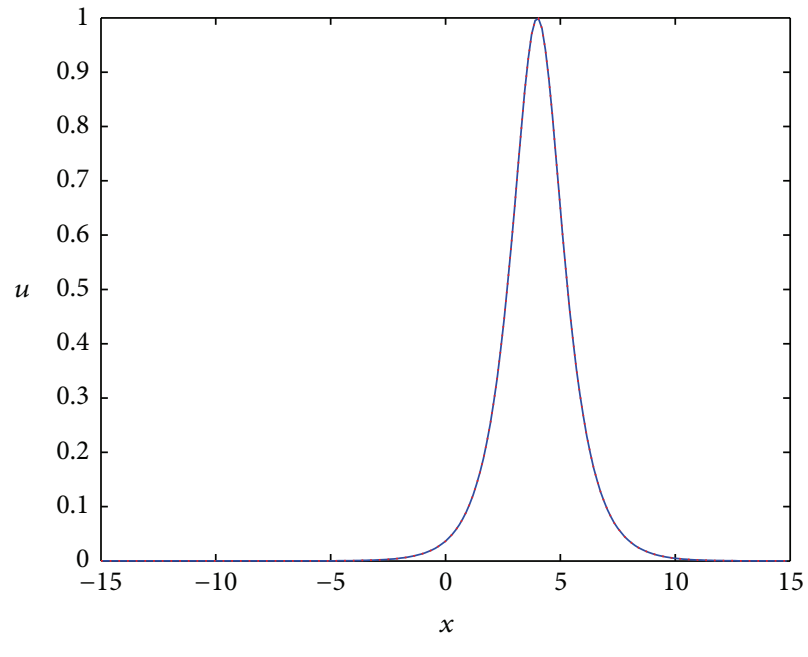

— Numerical solutions

(a) $t=1$

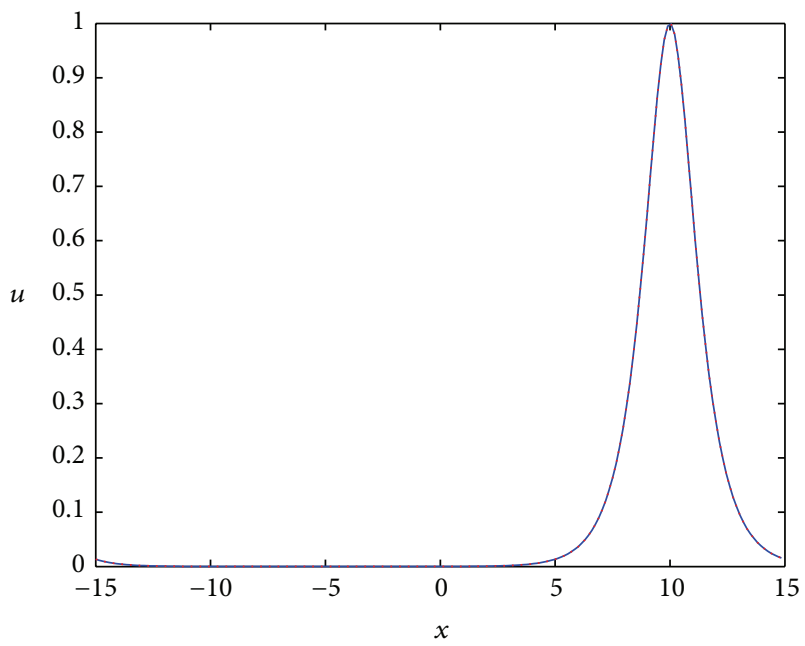

— Numerical solutions

(c) $t=10$

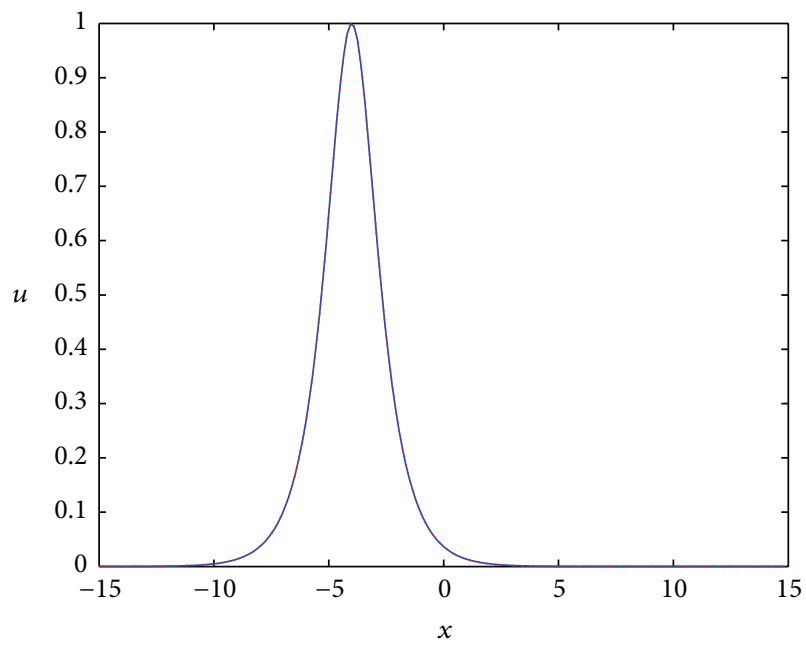

— Numerical solutions _..... Exact solutions

(e) $t=14$

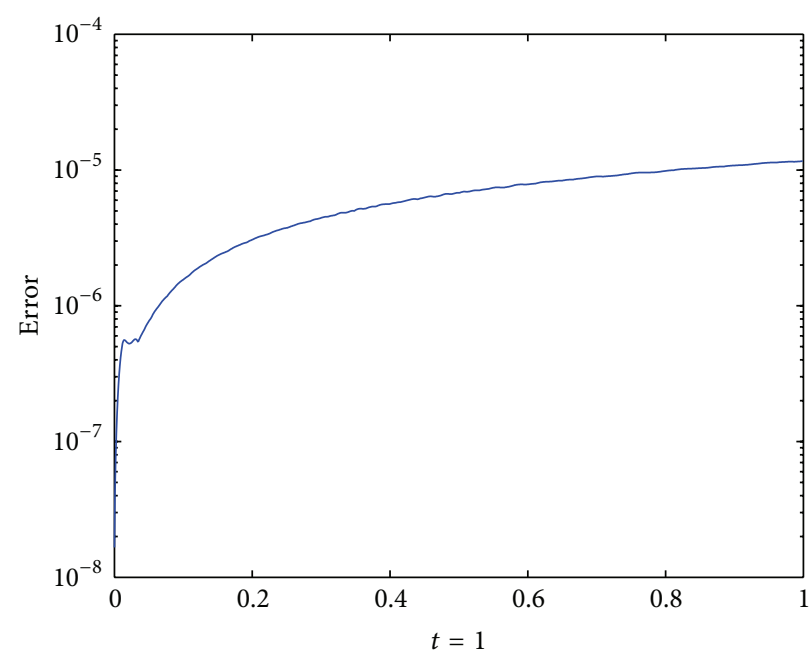

Errors between the exact solutions and the numerical solutions

(b) $0 \leq t \leq 1$

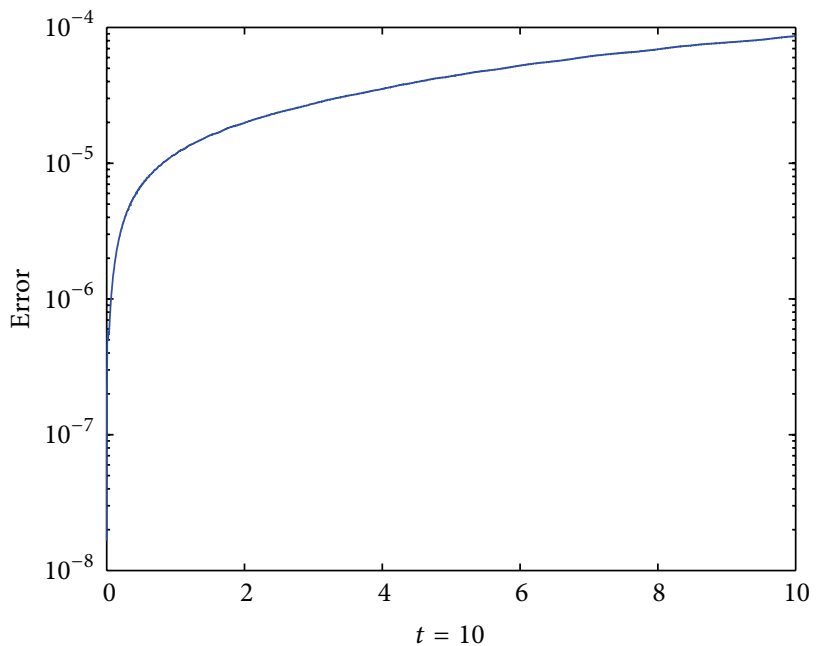

_ Errors between the exact solutions and the numerical solutions

(d) $0 \leq t \leq 10$

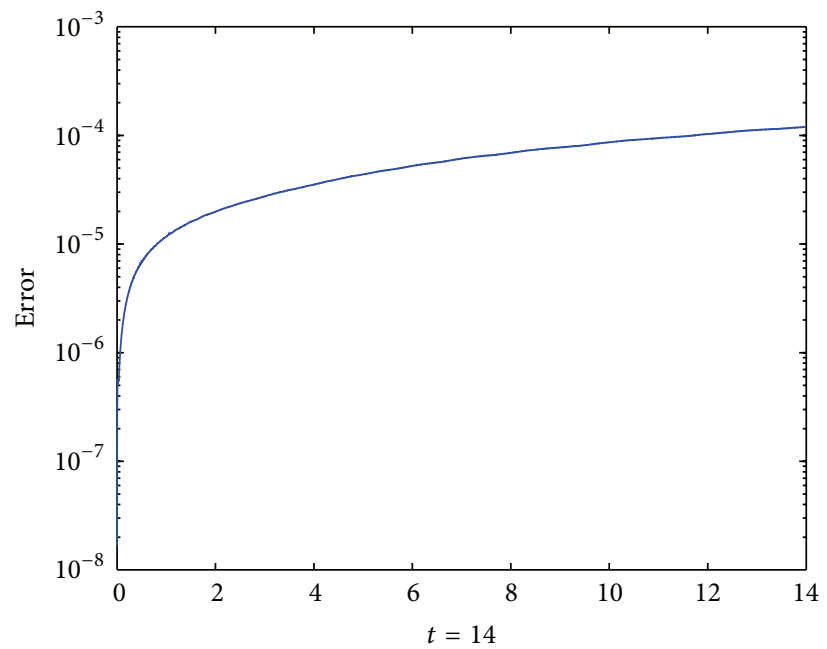

- Errors between the exact solutions and the numerical solutions

(f) $0 \leq t \leq 14$

Figure 1: Continued. 


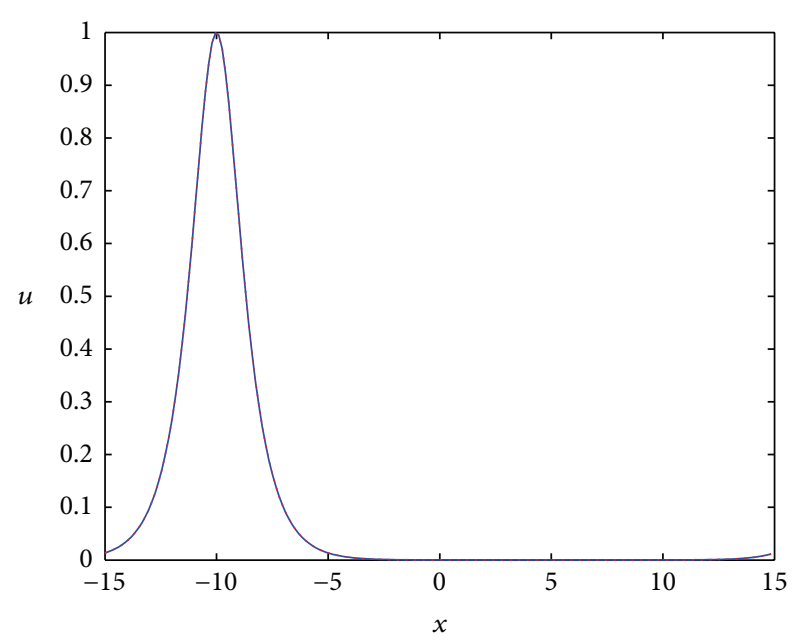

— Numerical solutions

(g) $t=50$

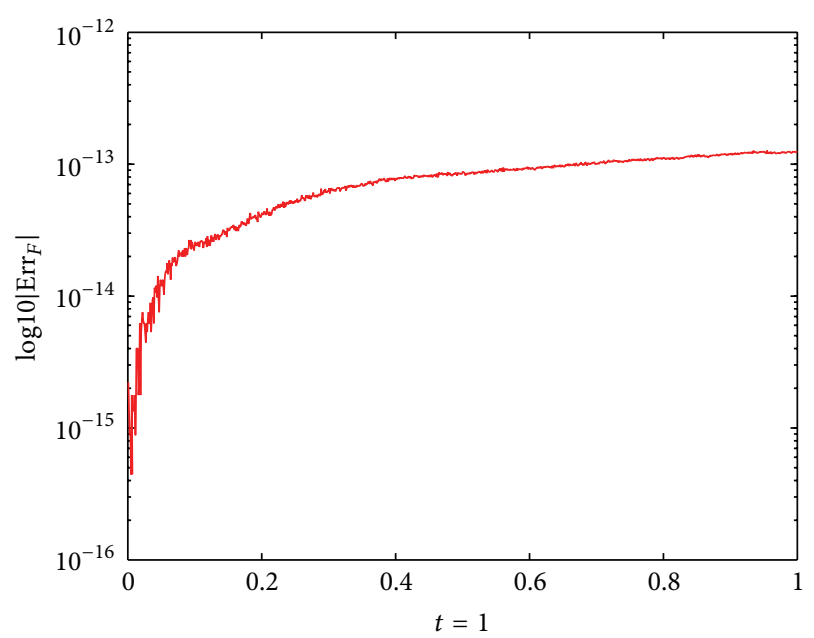

_ Errors of the discrete energy conservation law with time

(i) $0 \leq t \leq 1$

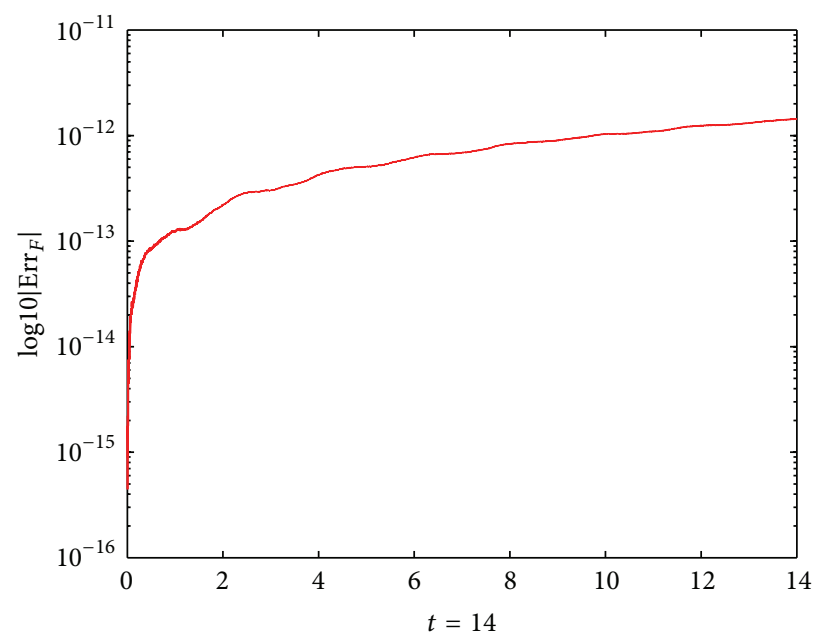

_ Errors of the discrete energy conservation law with time

(k) $0 \leq t \leq 14$

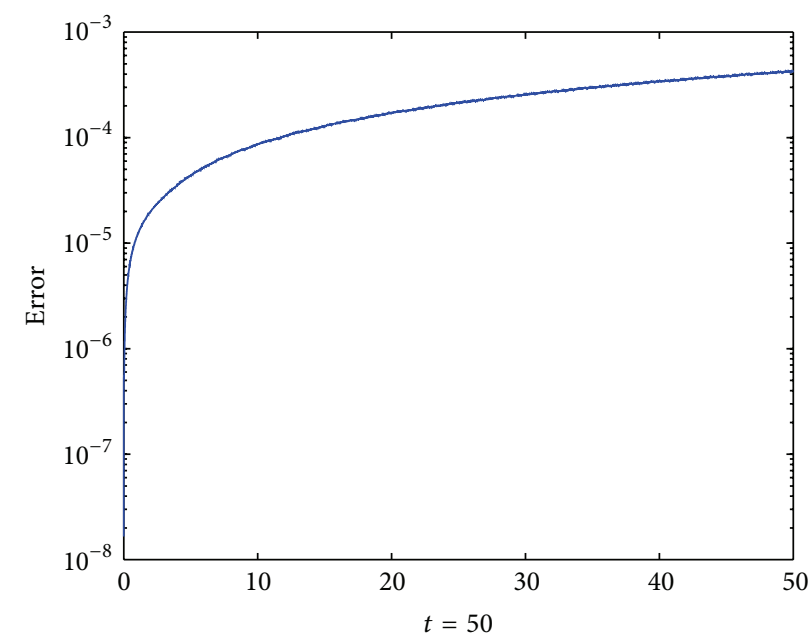

_ Errors between the exact solutions and the numerical solutions

(h) $0 \leq t \leq 50$

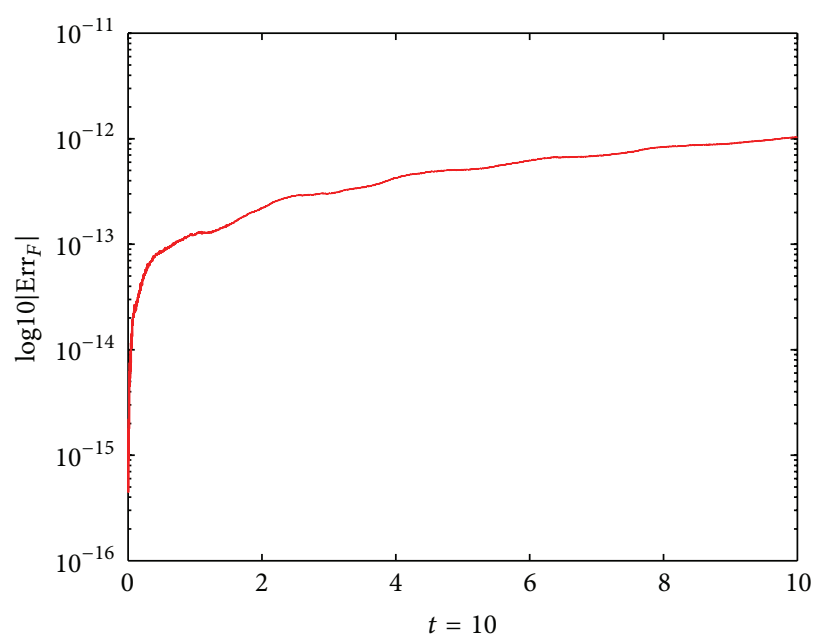

_ Errors of the discrete energy conservation law with time

(j) $0 \leq t \leq 10$

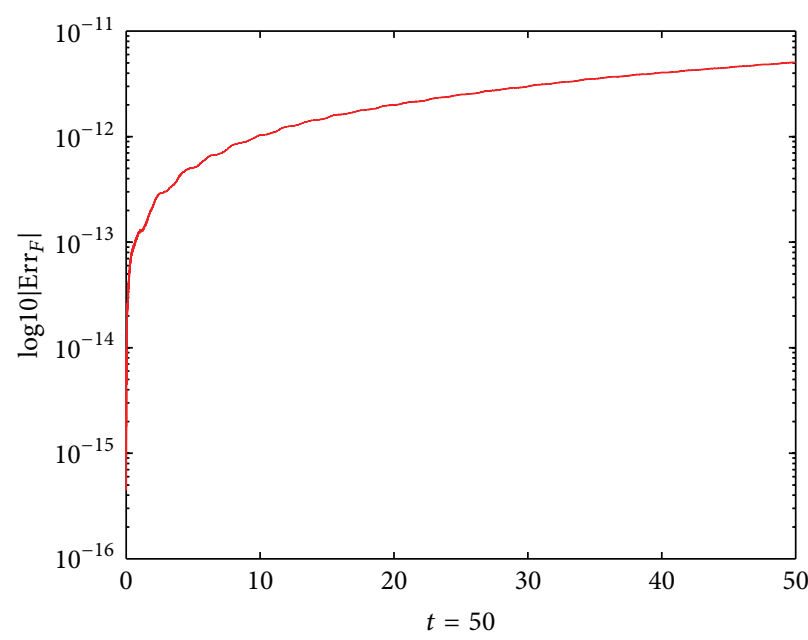

- Errors of the discrete energy conservation law with time

(l) $0 \leq t \leq 50$

FIGURE 1: The numerical solutions and the exact solutions at $t=1,10,14,50$ and the changes of the errors between the exact solutions and the numerical solutions and $\operatorname{Err}_{F}$ with time. 


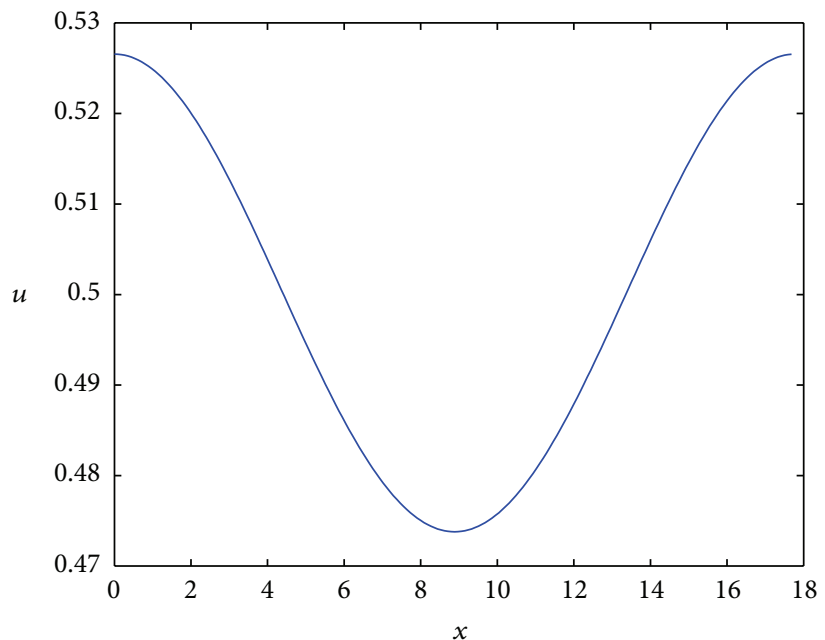

— Numerical solutions

(a) $t=1$

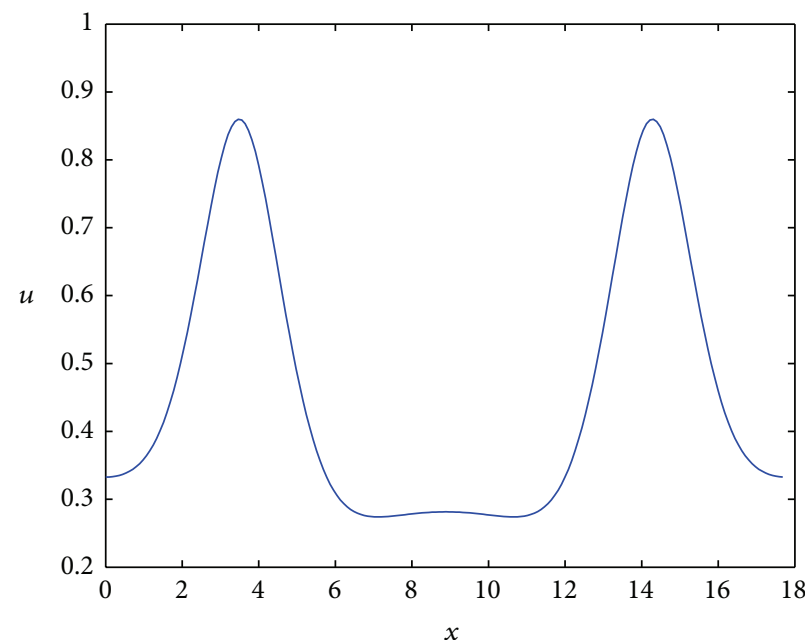

— Numerical solutions

(c) $t=14$

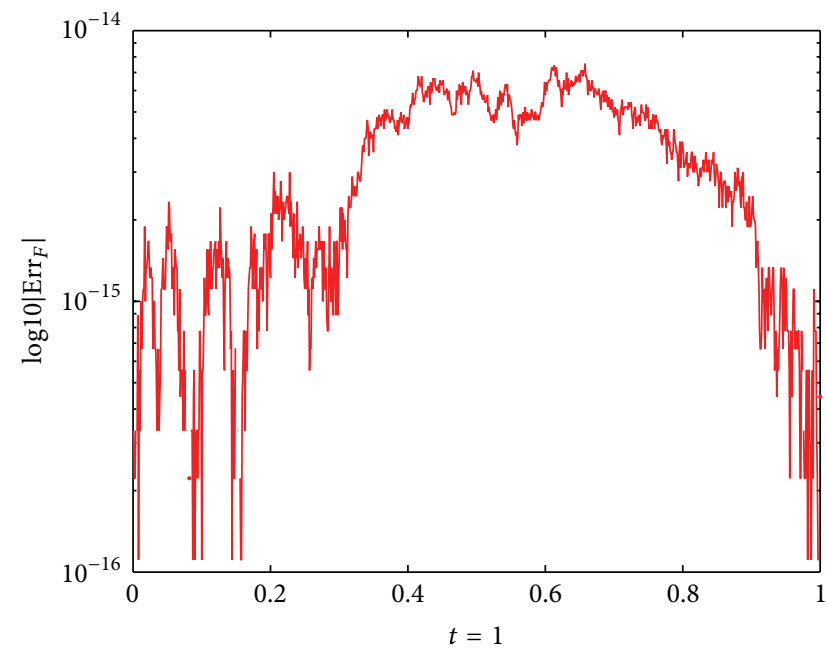

- Errors of the discrete energy conservation law with time

(e) $0 \leq t \leq 1$

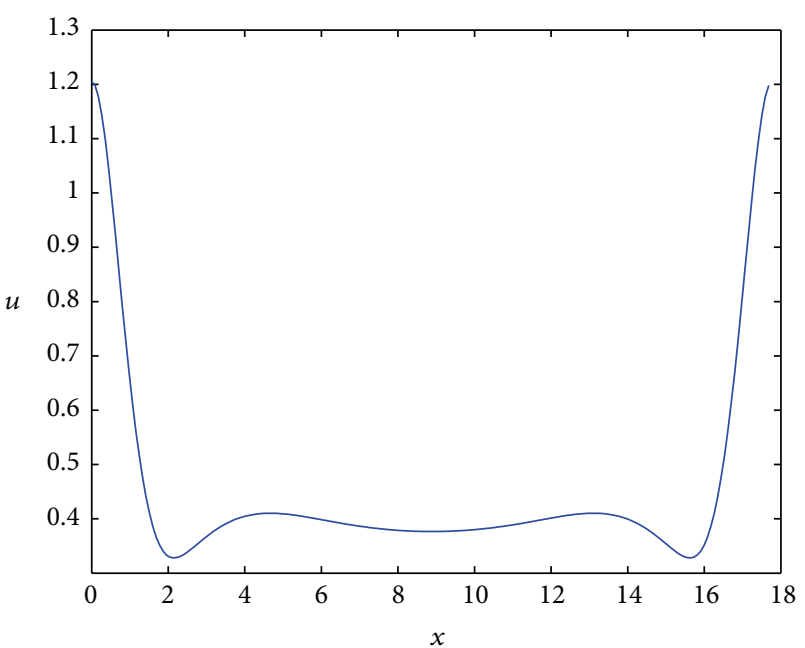

_ Numerical solutions

(b) $t=10$

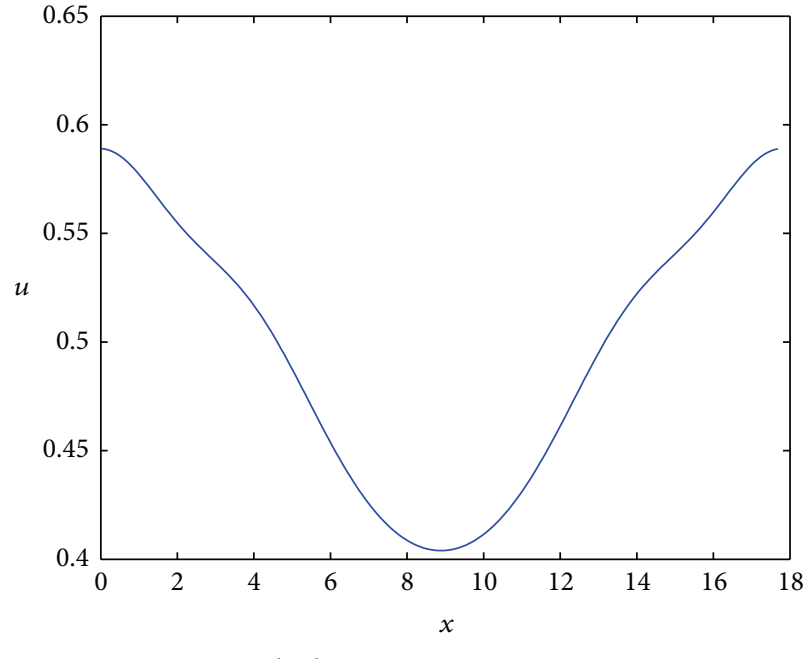

(d) $t=50$

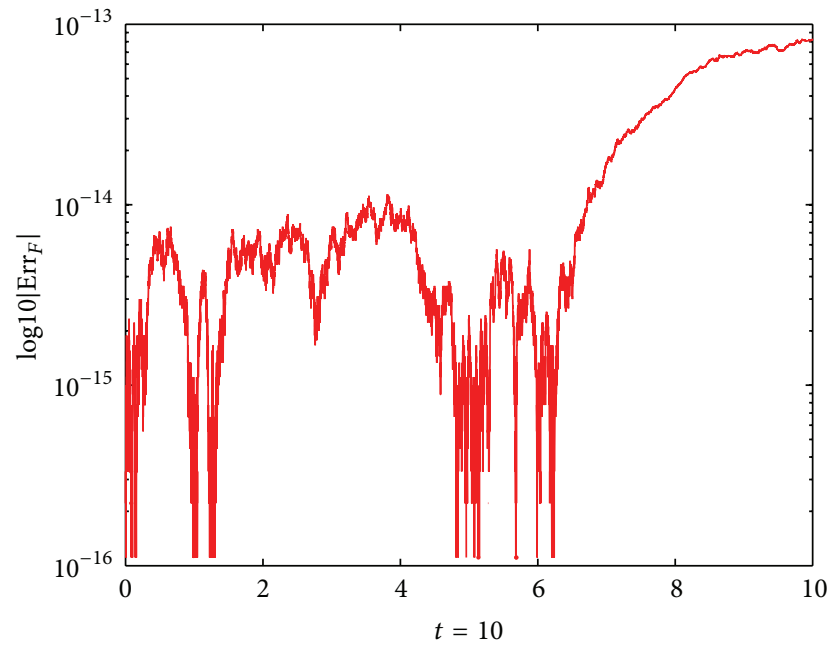

_ Errors of the discrete energy conservation law with time

(f) $0 \leq t \leq 10$

Figure 2: Continued. 


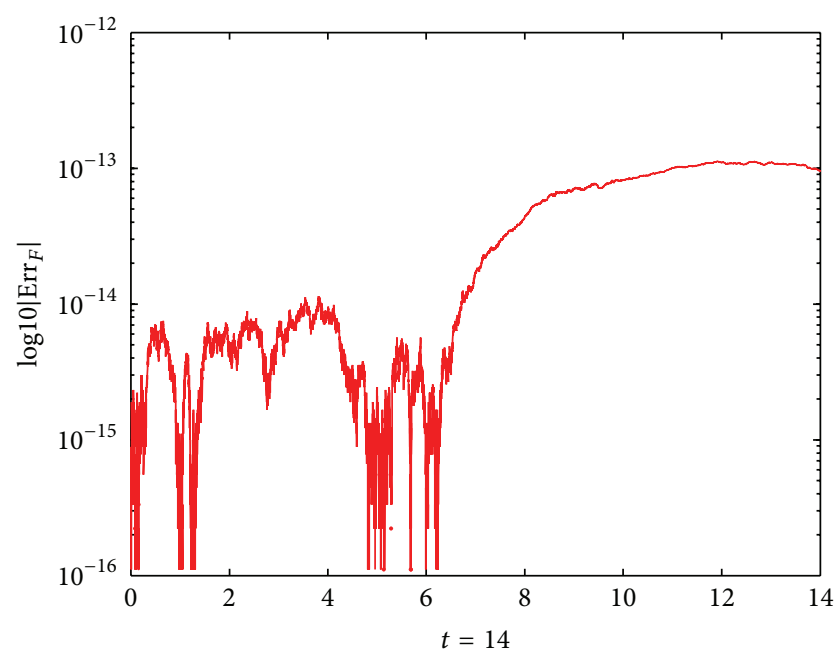

_ Errors of the discrete energy conservation law with time

(g) $0 \leq t \leq 14$

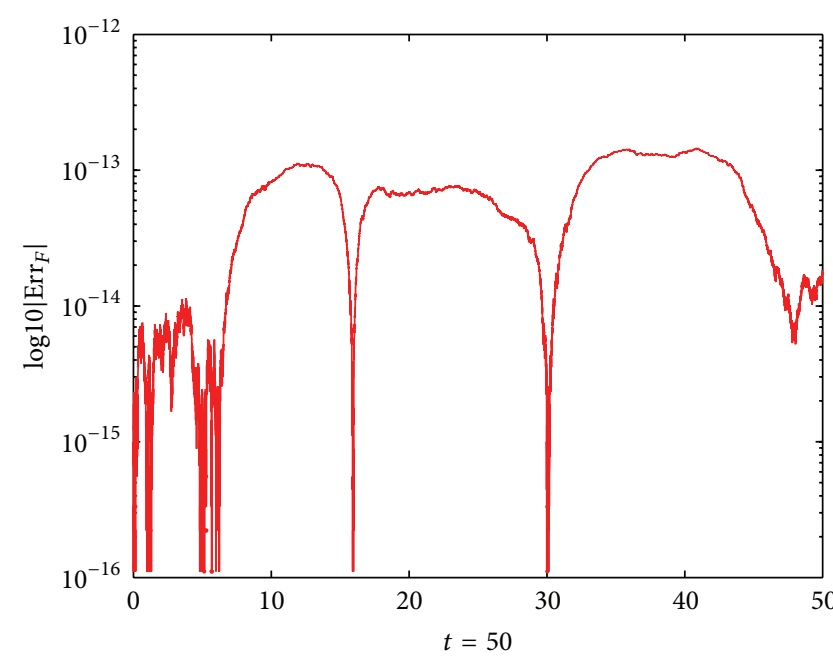

_ Errors of the discrete energy conservation law with time

(h) $0 \leq t \leq 50$

Figure 2: The numerical solutions at $t=1,10,14,50$ and the changes of $\operatorname{Err}_{F}$ with time.

\section{Conflict of Interests}

The authors declare that there is no conflict of interests regarding the publication of this paper.

\section{Acknowledgments}

This work is supported by the National Natural Science Foundation of China (Grants nos. 11271195, 41231173, and 11201169), the Postdoctoral Foundation of Jiangsu Province of China under Grant no. 1301030B, Open Fund Project of Jiangsu Key Laboratory for NSLSCS under Grant no. 201301, Fund Project for Highly Educated Talents of Nanjing Forestry University under Grant no. GXL201320, and the Project of Graduate Education Innovation of Jiangsu Province (Grant no. KYLX_0691).

\section{References}

[1] C. R. Menyuk, "Stability of solitons in birefringent optical fibers," Journal of the Optical Society of America B: Optical Physics, vol. 5, pp. 392-402, 1998.

[2] M. Wadati, T. Iizuka, and M. Hisakado, "A coupled nonlinear Schrödinger equation and optical solitons," Journal of the Physical Society of Japan, vol. 61, no. 7, pp. 2241-2245, 1992.

[3] K. Feng and M. Z. Qin, Symplectic Geometric Algorithms for Hamiltonian Systems, Springer, Hangzhou, China, 2010, (Chinese).

[4] M. Z. Qin and Y. S. Wang, Structure-Preserving Algorithm for PDEs, Zhejiang Press for Science and Technology, Hangzhou, China, 2011, (Chinese).

[5] J.-X. Cai and Y.-S. Wang, "A conservative Fourier pseudospectral algorithm for a coupled nonlinear Schrödinger system," Chinese Physics B, vol. 22, no. 6, Article ID 060207, 2013.

[6] G. D. Akrivis, "Finite difference discretization of the cubic Schrödinger equation," IMA Journal of Numerical Analysis, vol. 13, no. 1, pp. 115-124, 1993.
[7] C.-L. Jiang and J.-Q. Sun, "A high order energy preserving scheme for the strongly coupled nonlinear Schrödinger system," Chinese Physics B, vol. 23, no. 5, Article ID 050202, 2014.

[8] Z.-Q. Lv, Y.-S. Wang, and Y.-Z. Song, "A new multi-symplectic integration method for the nonlinear schrödinger equation," Chinese Physics Letters, vol. 30, no. 3, Article ID 030201, 2013.

[9] M. Dehghan and A. Taleei, "A compact split-step finite difference method for solving the nonlinear Schrödinger equations with constant and variable coefficients," Computer Physics Communications, vol. 181, no. 1, pp. 43-51, 2010.

[10] Q. Chang, E. Jia, and W. Sun, "Difference schemes for solving the generalized nonlinear Schrödinger equation," Journal of Computational Physics, vol. 148, no. 2, pp. 397-415, 1999.

[11] T. C. Wang and B. L. Guo, "Unconditional convergence of two conservative compact difference schemes for non-linear Schrödinger equation in one dimension," Science in China Series A: Mathematics, vol. 41, no. 3, pp. 207-233, 2011.

[12] Y. S. Wang, Q. H. Li, and Y. Z. Song, "Two new simple multisymplectic schemes for the nonlinear Schrödinger equation," Chinese Physics Letters, vol. 25, no. 5, pp. 1538-1540, 2008.

[13] Y. S. Wang and S. T. Li, "New schemes for the coupled nonlinear Schrödinger equation," International Journal of Computer Mathematics, vol. 87, no. 4, pp. 775-787, 2010.

[14] R.-P. Zhang, X.-J. Yu, and T. Feng, "Solving coupled nonlinear Schrödinger equations via a direct discontinuous Galerkin method," Chinese Physics B, vol. 21, no. 3, Article ID 030202, 2012.

[15] G. R. Quispel and D. I. McLaren, "A new class of energypreserving numerical integration methods," Journal of Physics A: Mathematical and Theoretical, vol. 41, no. 4, Article ID 045206, 7 pages, 2008.

[16] E. Celledoni, V. Grimm, R. I. McLachlan et al., "Preserving energy resp. dissipation in numerical PDEs using the 'average vector field' method," Journal of Computational Physics, vol. 231, no. 20, pp. 6770-6789, 2012.

[17] C. Canuto and A. Quarteroni, "Approximation results for orthogonal polynomials in Sobolev spaces," Mathematics of Computation, vol. 38, no. 157, pp. 67-86, 1982. 
[18] Y. L. Zhou, Application of Discrete Functional Analysis to the Finite Diserence Methods, International Academic Publishers, Beijing, China, 1990.

[19] J.-B. Chen and M.-Z. Qin, "Multi-symplectic Fourier pseudospectral method for the nonlinear Schrödinger equation," Electronic Transactions on Numerical Analysis, vol. 12, pp. 193204, 2001. 


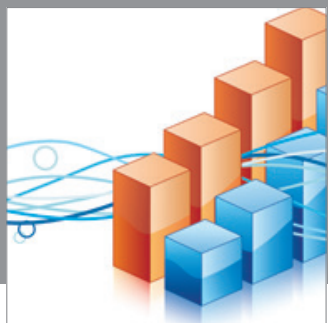

Advances in

Operations Research

mansans

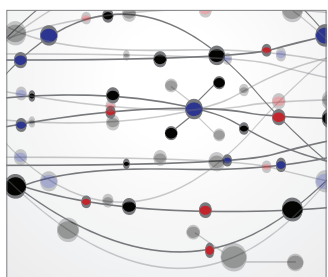

The Scientific World Journal
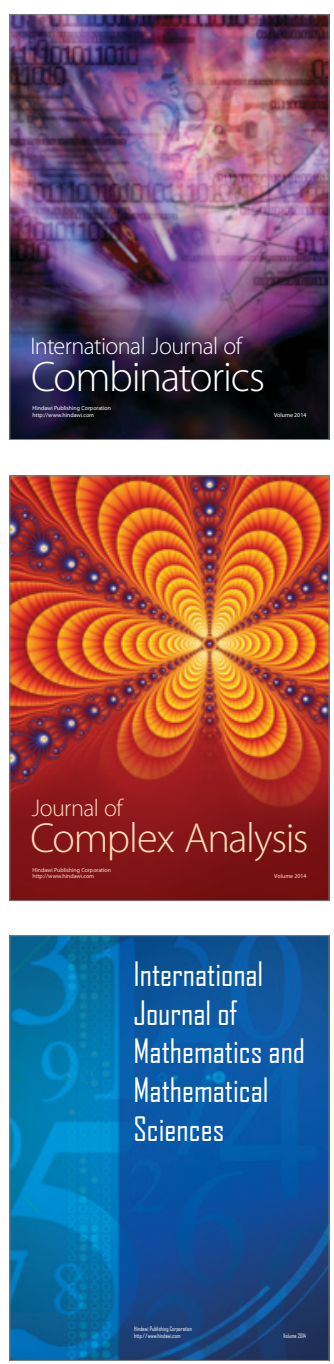
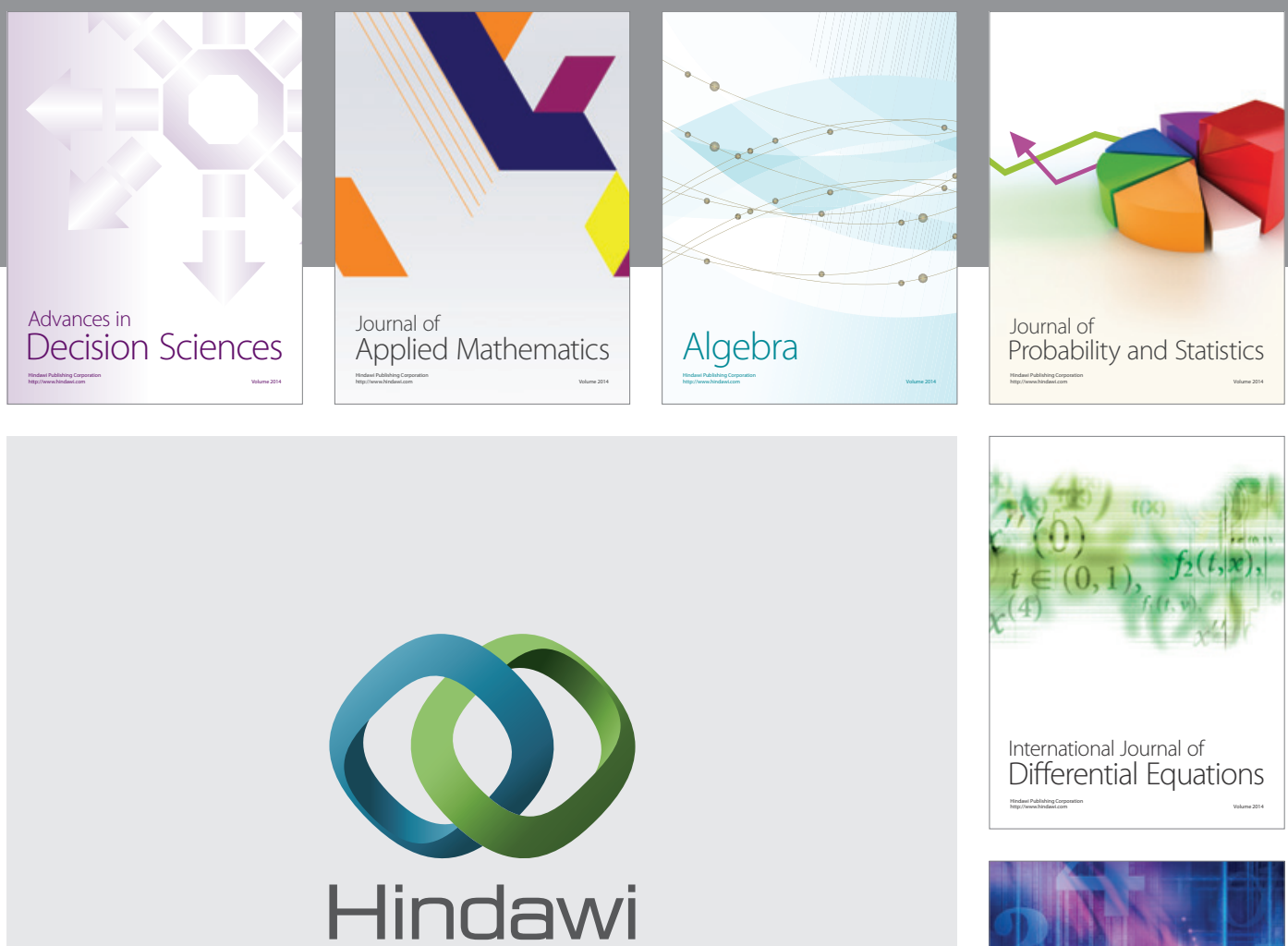

Submit your manuscripts at http://www.hindawi.com
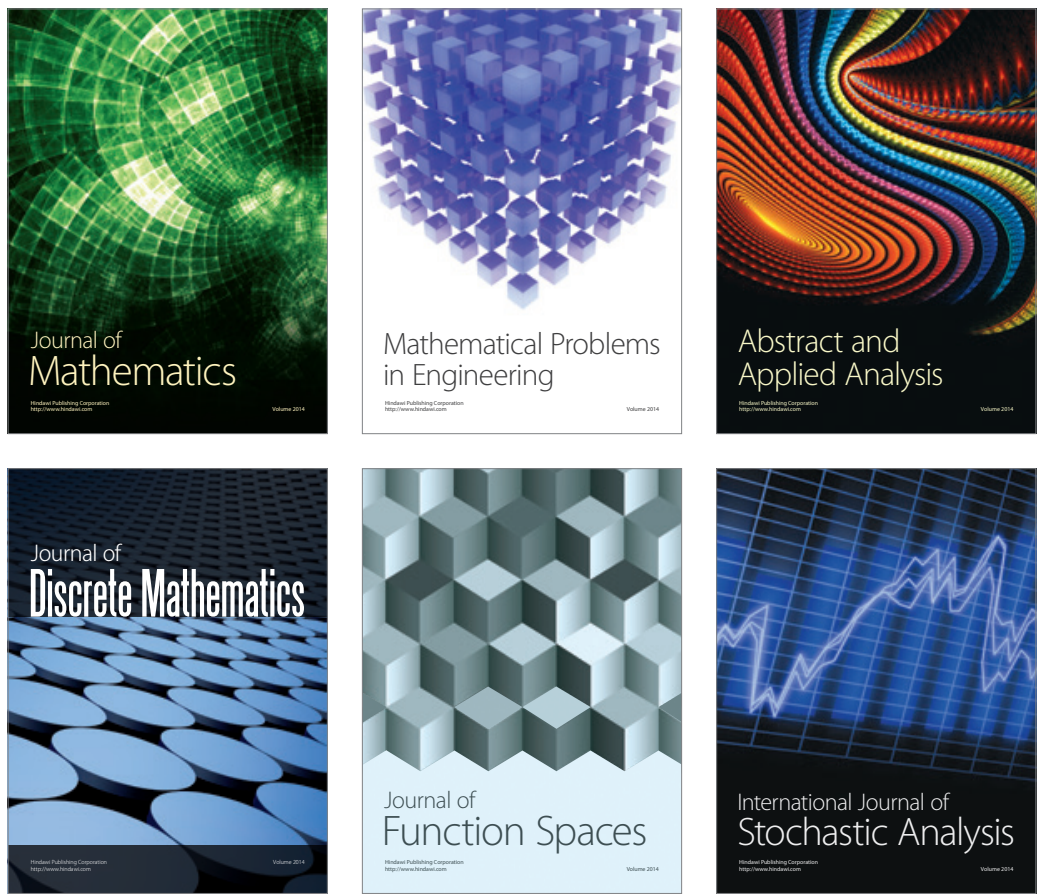

Journal of

Function Spaces

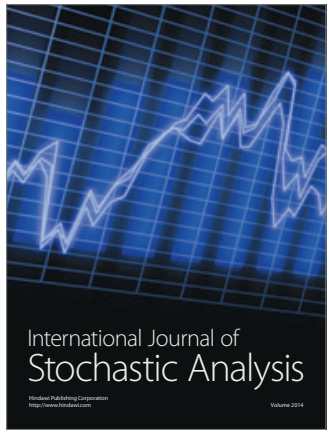

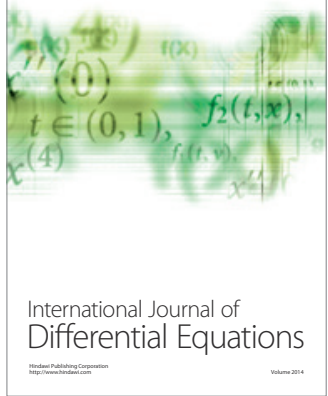
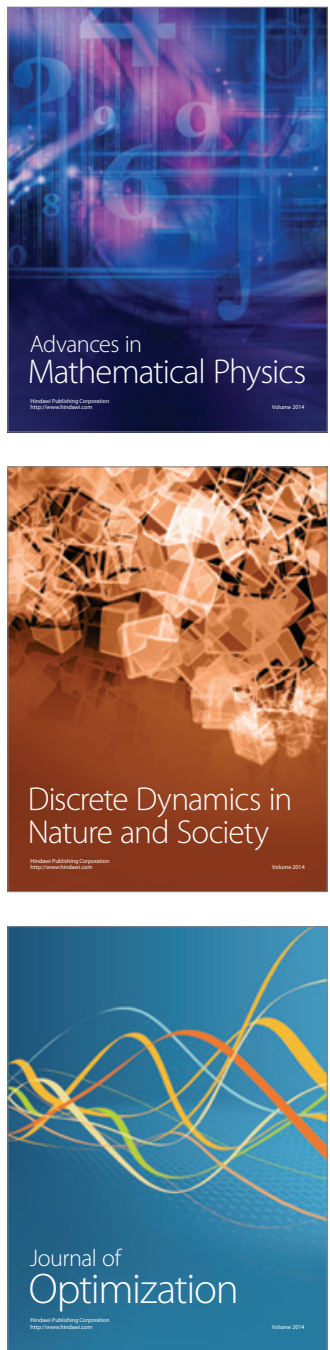